\title{
The Feasibility of Platelet-Rich Fibrin Matrix Filler in a Nude Mouse Model
}

Hyeon Jun Jeon ${ }^{1}$, Dong Kyu Kim ${ }^{1}$, Jeong Woo Lee ${ }^{1}$, Kang Young Choi ${ }^{1}$, Ho Yun Chung ${ }^{1}$, Byung Chae Cho ${ }^{1}$, Jun Ho Shin'2, Seung Ryul Lee ${ }^{3}$, Jung Dug Yang ${ }^{1}$

${ }^{1}$ Department of Plastic and Reconstructive Surgery, Kyungpook National University School of Medicine, Daegu; ${ }^{2}$ Urban Beauty Plastic Surgery, Seoul; ${ }^{3}$ Plus Plastic Surgery, Daejeon, Korea

No potential conflict of interest relevant to this article was reported.
Background In contrast to fillers made from artificial substances, platelet-rich fibrin matrix (PRFM) filler does not cause hypersensitivity reactions or foreign body reactions. PRFM is also highly accessible in terms of cost. Hence, in this study, the efficacy of PRFM for soft tissue augmentation and volume maintenance was evaluated in an animal experiment.

Methods Twenty nude mice were injected with hyaluronic acid filler, fibrin glue, PRFM filler, and normal saline (control). The remaining volume was measured 4 times over the course of 8 weeks using the volumetric taping bowl method and magnetic resonance imaging.

Results All nude mice survived and showed no signs of infection, such as erythema or edematous changes, during the study period. Migration of the injected substance was not detected at 2, 4, or 8 weeks after the procedure. The remaining volumes of normal saline at 2, 4, and 8 weeks were $10.50 \%, 2.00 \%$, and $0.00 \%$; fibrin glue, $20.50 \%, 9.00 \%$, and $2.50 \%$; hyaluronic acid filler, $82.00 \%, 35.00 \%$, and $17.33 \%$; and PRFM filler, $70.31 \%, 26.75 \%$, and $14.37 \%$, respectively.

Conclusions PRFM filler had a high soft-tissue filling capacity compared with the control. It also showed a similar effect to hyaluronic acid filler. Thus, PRFM filler could be a good alternative for correcting soft-tissue deficits.

Keywords Cosmetic techniques, Fibrin, Hyaluronic acid, Hypersensitivity, Platelet-rich plasma

\section{INTRODUCTION}

With aging, the face develops numerous wrinkles and can acquire deformities, such as soft-tissue deficits, due to trauma. Over the last 30 years, facial rejuvenation for the correction of facial wrinkles or small deficits has developed dramatically from the treatment of superficial rhytides, focusing on methods of revolumization. These developments stem from a comprehensive understanding of the changes in the bone and soft tissue that can occur in an aging face.

Received: Sep 1, 2017 Revised: Oct 10, 2017 Accepted: Oct 10, 2017 Correspondence: Jung Dug Yang Department of Plastic and Reconstructive Surgery, Kyungpook National University School of Medicine, 130 Dongdeokro, Jung-gu, Daegu 41944, Korea. E-mail: lambyang@knu.ac.kr

Copyright @ 2017 The Korean Society for Aesthetic Plastic Surgery.

This is an Open Access article distributed under the terms of the Creative Commons Attribution Non-Commercial License (http://creativecommons.org/licenses/by-nc/4.0/) which permits unrestricted non-commercial use, distribution, and reproduction in any medium, provided the original work is properly cited. $\quad w w w . e-a a p s . o r g$
Recently, with increasing interest in beauty, a number of researchers have been performing wrinkle or facial correction using lowcost, simple procedures with rapid recovery times. Due to these trends, various types of filler are being researched and developed, and the range of procedures in which they are applied is becoming broader. Over the last several years, injectable fillers have become essential in cosmetic therapy. These advances are ongoing, and we are coming closer to finding an ideal product that is durable, permanent, and well tolerated. In addition, such fillers could provide subtle, natural, and potentially reversible outcomes.

Dermal stimulation and augmentation are fundamental facial treatments in the field of cosmetics, and they are being used increasingly often. These treatments initially use bioresorbable substances, such as hyaluronic acid [1]. Many exogenous fillers show volume augmentation while maintaining a fibrotic response at the dermal level [2]. However, despite the biodegradability of the substances, exogenous injections may show obvious adverse effects. These include transient effects, such as persistent erythema, swell- 
ing, encapsulation, and granuloma formation, as well as chronic or delayed infections [3-6]. For this reason, doctors have been searching for autologous sources for soft tissue augmentation. Autologous platelets are an excellent source of growth factors due to their biological properties and endogenous origin. They are currently the main source of growth factors that promote wound healing. Numerous studies have shown that platelet-rich plasma (PRP) promoted early wound healing [7-9] and was effective at enhancing the treatment of diabetic ulcers [10]. PRP is a desirable autologous source to promote soft-tissue deposition in areas of depletion, and it is often used in oral, maxillofacial, and plastic surgery procedures. As a natural reservoir of growth factors, PRP can be used to facilitate the synthesis of collagen in fibroblasts, keratinocyte proliferation, and hyaluronic acid generation, thereby increasing dermal elasticity and producing a positive effect on facial rejuvenation $[11,12]$. Because platelet-rich fibrin matrix (PRFM) filler is isolated from autologous blood, it is safe from immune rejection, but many conflicting opinions exist about its long-term use. Thus, in this study, Thrombo, Push-Man, and Air-Man kits (Melsmon, Seongnam, Korea) were used to prepare PRFM. An animal experiment was conducted using nude mice to examine the efficacy of PRFM filler containing autologous plasma on soft tissue augmentation and volume maintenance.

\section{METHODS}

\section{Materials}

Twenty nude mice weighing 200 to $300 \mathrm{~g}$ were raised by a breeder at a constant temperature and humidity. Hyaluronic acid filler (Restylane ${ }^{\circledR}$; Q-Med, Uppsala, Sweden), PRFM filler from autologous plasma, fibrin glue (Tissel ${ }^{\circledR}$; Baxter AG, Vienna, Austria), and phys- iological saline were used. Four locations on the back of the 2 nude mice were prepared and injected with $1 \mathrm{~mL}$ of each of the 4 fillers: normal saline (location A), fibrin glue (location B), hyaluronic acid filler (location C), and PRFM (location D).

\section{Methods}

\section{Fabrication of PRFM filler}

Thrombo, Push-Man, and Air-Man kits were used to prepare PRFM filler before injection. To prevent coagulation, $2 \mathrm{~mL}$ of heparin was added to $8 \mathrm{~mL}$ of blood in a $10-\mathrm{mL}$ syringe, and the $10-\mathrm{mL}$ heparin-blood mixture was injected into the Thrombo kit. Before centrifugation, the Thrombo kit was weighed to prepare another Thrombo kit containing saline with the same weight, and the 2 tubes were placed in opposite positions in the centrifuge (Fig. 1). After centrifugation for 6 minutes at 2,000 g, the Thrombo kit was removed and inserted into the Push-Man kit. The centrifuged blood contained platelet-deficient plasma, buffy coat, and erythrocyte layers. The platelet-deficient plasma and buffy coat layers were then transferred to a 1-mL syringe using the Push-Man kit to prepare PRP. Subsequently, the PRP was heated at $91^{\circ} \mathrm{C}$ using the Air-Man kit for 10 minutes and then cooled at $4^{\circ} \mathrm{C}$ for 5 minutes for solidification. The solidified PRP was stored at a consistent temperature of $4^{\circ} \mathrm{C}$ until use.

\section{Injection of PRFM filler}

The 20 mice were divided into groups of 2 , and 2 injection sites were drawn on the back of each mouse for a total of 40 injection. Normal saline, fibrin glue, hyaluronic acid filler, and PRFM filler were injected into each site. Anesthesia was maintained with xylazine $\mathrm{HCl}$ (Rompun ${ }^{\circledR}$, Bayer Korea Ltd., Seoul, Korea) (10 mg/kg) and ketamine $\mathrm{HCl}$ (Huons ketamine- $\mathrm{HCl}^{\circledR}$; Huons, Seongnam,
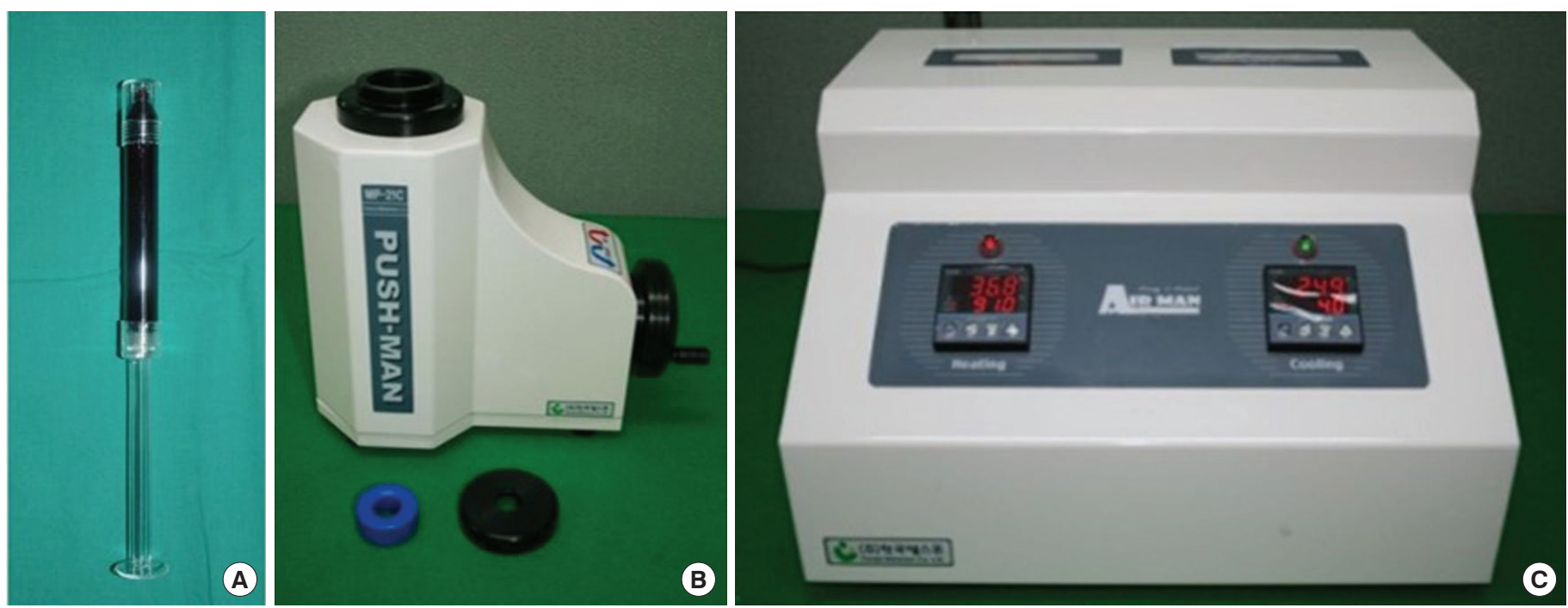

Fig. 1. Gross photograph of the platelet-rich fibrin matrix (PRFM) filler system. The PRFM filler system was composed of (A) a Thrombo kit (Melsmon, Seongnam, Korea), (B) Push-Man kit (Melsmon), and an (C) Air-Man kit (Melsmon). 
Korea) $(100 \mathrm{mg} / \mathrm{kg})$ via an intra-abdominal injection. With the mice in the prone position, the abdominal region was disinfected with betadine and alcohol, and a 30-gauge syringe was used to intradermally inject the prepared fillers evenly into the 20 mice, such that all 4 fillers were injected into a group of 2 mice (Fig. 2). All fillers were stored in a refrigerator until injection, and the nude mice were housed at room temperature. To prevent infection, the injection sites were dressed daily until postoperative day 7 , but antibiotics were not used. The tissues surrounding the injection site were isolated at 4 and 8 weeks after filler injection.

\section{Assessment \\ Volumetry}

The volume of the injected filler was measured using the volumetric taping bowl method and magnetic resonance imaging (MRI) (3.0 Tesla System; GE Healthcare, Milwaukee, WI, USA) under intra-abdominal anesthesia on the day of the experiment and at weeks 2,4 , and 8 after the experiment. The measurements using the taping bowl method were performed by 3 different doctors, 3 times each, using an identical method. Hence, a total of 9 measurements were obtained and averaged. The average of the first set of measurements of residual filler volume using the volumetric taping bowl method and the second set of measurements of residual filler volume using the MRI system was used as the final residual filler volume.

Volumetry using a volumetric taping bowl

The margins of the fillers injected into the soft tissues were identified, and taping was performed precisely using 8 tapes (length, 1
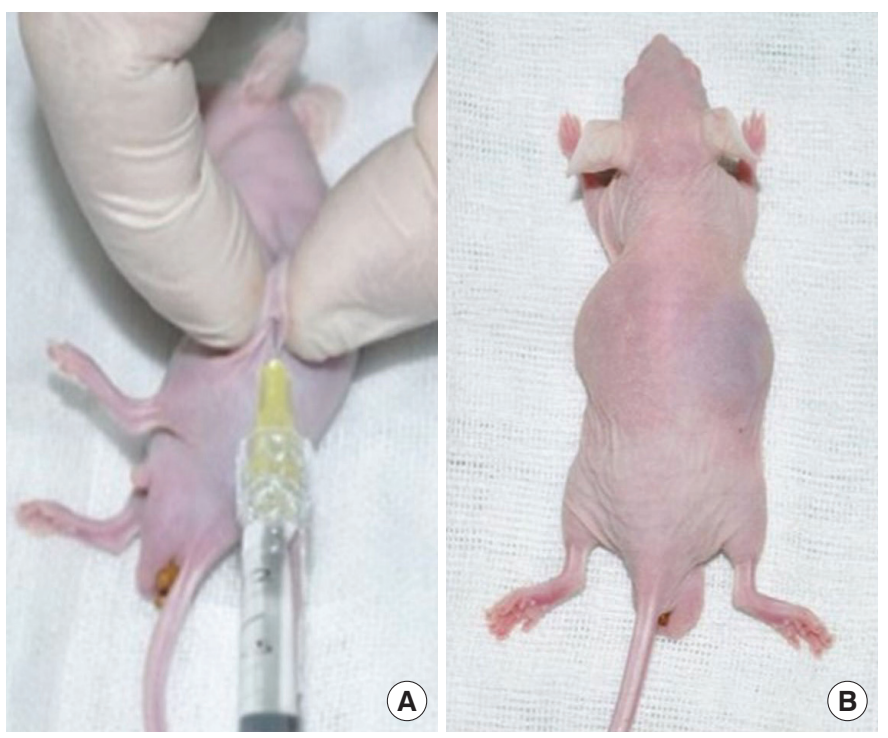

Fig. 2. Gross photograph of the intradermal filler injection. (A) Four types of filler were injected in each mouse intradermally. (B) Gross photograph of the injection area after the procedure. $\mathrm{cm}$; width, $0.5 \mathrm{~cm}$ ) per measurement area to make a volumetric taping bowl. The tapes were removed to create a volumetric taping bowl corresponding to the volume of the residual filler. Normal saline was micropipetted into the volumetric taping bowl to measure the exact volume of the residual filler (Fig. 3).

Volumetry using MRI

After measuring the volume using a volumetric taping bowl, MRI of the filler injection site was performed. The MRI images were then converted into 3-dimensional images using the volume rendering program of the Advanced Workstation Volume Share Version 4.5 System (GE Healthcare), which was then used to quantify the volume with the volume measuring tool (Fig. 4).

\section{Histological assessment}

Residual fillers and the surrounding capsule were extracted from the 20 nude mice at postoperative weeks 4 and 8 and were fixed with $10 \%$ neutral formalin to acquire 20 tissue samples. After being embedded in paraffin, tissue slices were made and stained with hematoxylin and eosin and Masson trichrome. The stained slices were then observed under a light microscope (Nikon ECLIPSE 80i; Nikon Corporation, Tokyo, Japan) to assess the inflammatory response, reactions with the surrounding tissue, and new collagen fiber formation. Filler absorption was examined and compared based on the density and compactness of the tissue at high magnification.
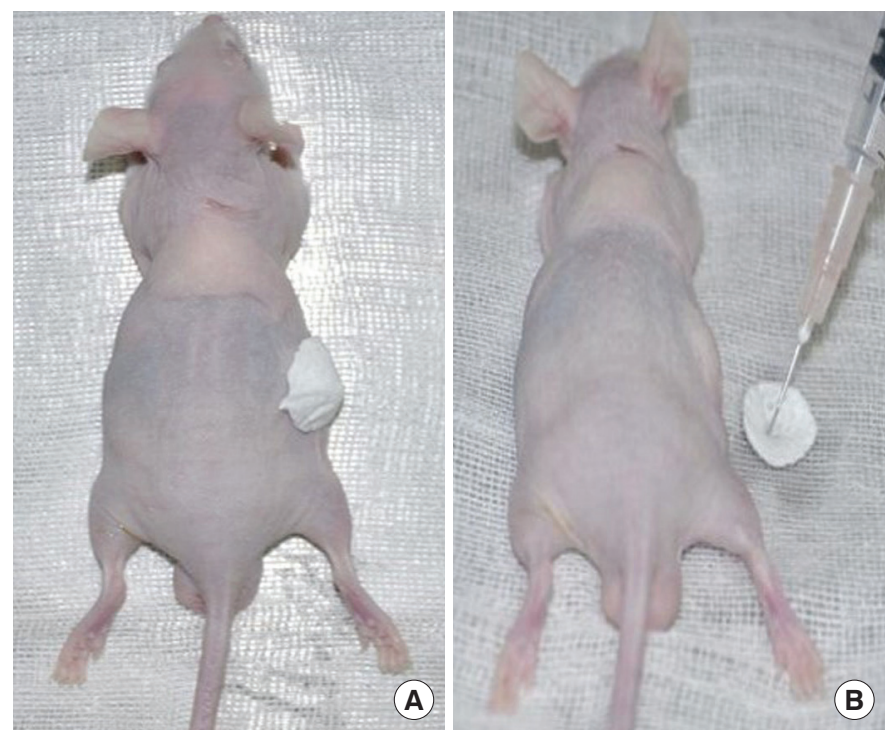

Fig. 3. Procedure for making a volumetric taping bowl. (A) Pliable, sticky medical tape was used to make a volumetric taping bowl, and the volume of the residual filler was closely related to the size of the bowl. (B) The residual volume of the filler was evaluated 4 times over the course of 8 weeks. The volume of saline in the bowl was examined using a syringe and a micropipette. 

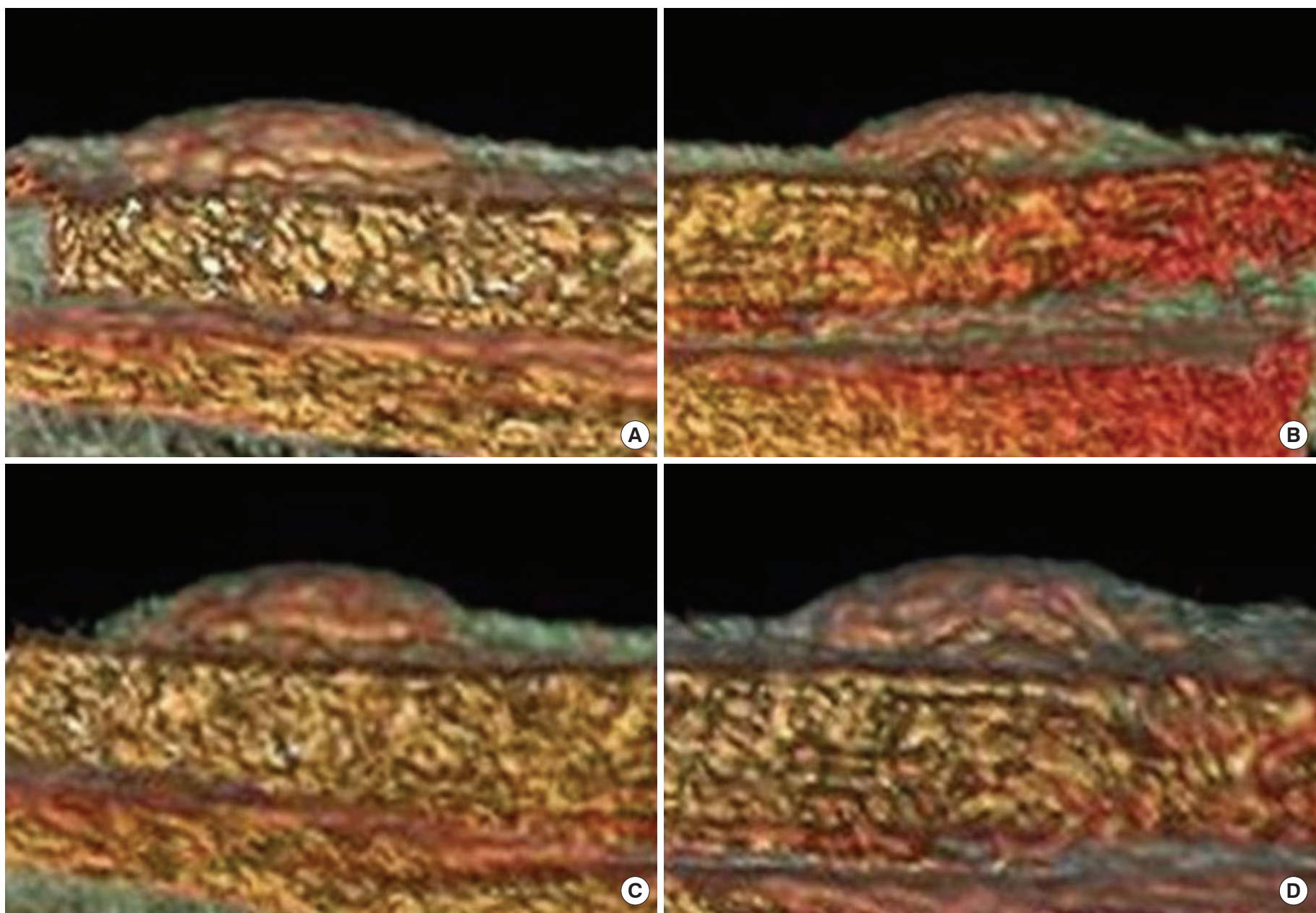

Fig. 4. Three-dimensional reconstructed image of the filler injection area obtained using magnetic resonance imaging at 4 weeks after the injection of 4 types of filler. (A) Normal saline, (B) fibrin glue, (C) hyaluronic acid filler, and (D) platelet-rich fibrin matrix filler.

\section{Statistical analysis}

All measured data were statistically analyzed using SPSS version 16.0 (SPSS Inc., Chicago, IL, USA). The residual filler volume among the groups was compared using the Kruskal-Wallis test. Post-hoc tests of the average of each group's residual volume were performed using Dunn multiple comparison. P-values $<0.05$ were considered to indicate statistical significance.

\section{RESULTS}

All nude mice survived the study period. Leakage or migration of injected filler material and inflammation were not observed in any of the mice.

\section{Gross findings}

Inflammatory findings, such as flare-ups or edema in the injection sites, and filler migration were not observed throughout the 8-week study. The hyaluronic acid and PRFM fillers, which were relatively solid, formed regular nodules upon injection, whereas the normal saline and fibrin glue, which were in liquid form, created broader nodules than the solidified materials. Furthermore, the hyaluronic acid fillers were more easily manipulated to achieve the desired shape than the PRFM fillers, but these 2 types of filler did not differ in their effects on the augmentation of soft tissues at the injection site.

\section{Volumetry}

Volumes were measured 4 times over the course of 8 weeks: on the day of injection and at weeks 2,4 , and 8 after the experiment. At week 2 , the residual volumes compared to the initial injection volumes were $10.50 \%(0.105 \mathrm{~mL})$ for normal saline, $20.50 \%$ (0.205 $\mathrm{mL}$ ) for fibrin glue, $70.31 \%(0.703 \mathrm{~mL})$ for the PRFM filler, and $82.00 \%(0.820 \mathrm{~mL})$ for the hyaluronic acid filler. This result indicates that the normal saline and fibrin glue were mostly absorbed, whereas much of the initial injection volume was maintained for the PRFM and hyaluronic acid fillers, with only a small amount absorbed. At week 4, normal saline and fibrin glue showed a marked 


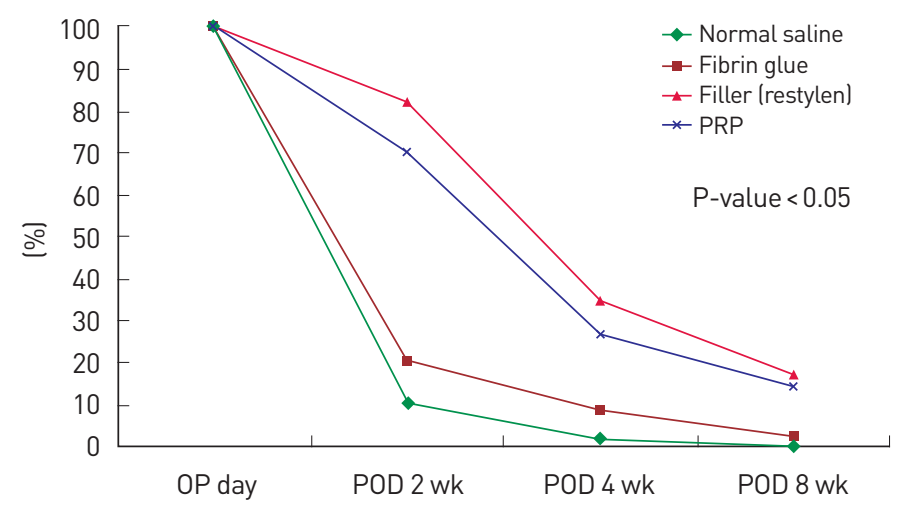

Fig. 5. Residual volume graph of the 4 types of filler. The remaining volumes of the hyaluronic acid and platelet-rich fibrin matrix (PRFM) fillers were higher than those of normal saline and fibrin glue at 4 and 8 weeks after the experiment. Compared with the hyaluronic acid filler, the PRFM filler did not show a significant difference in soft tissue filling capacity or durability at 2, 4, or 8 weeks after the procedure $(P<0.05)$. PRP, platelet-rich plasma; OP, operative; POD, postoperative day; wk, weeks.

decrease in volume, with residual volumes of $2.00 \%(0.020 \mathrm{~mL})$ and $9.00 \%(0.090 \mathrm{~mL})$, respectively. In contrast, the PRFM and hyaluronic acid fillers were relatively well maintained, with residual volumes of $26.75 \%(0.267 \mathrm{~mL})$ and $35.00 \%(0.350 \mathrm{~mL})$, respectively. At week 8 , the normal saline had been completely absorbed, and $2.50 \%(0.025 \mathrm{~mL})$ of fibrin glue remained; the PRFM and hyaluronic acid fillers were relatively well maintained, with volumes of $14.37 \%$ $(0.143 \mathrm{~mL})$ and $17.33 \%(0.173 \mathrm{~mL})$, respectively. No statistically significant differences were found in the volumes of hyaluronic acid and PRFM fillers at any of the 4 different measurement points throughout the 8 weeks of the study period $(\mathrm{P}<0.05)$ (Fig. 5 and Table 1).

\section{Histological findings Inflammatory cell count}

To examine the extent of the inflammatory reaction after filler injection, areas with a high density of inflammatory cells were randomly chosen and polymorphonuclear leukocytes and monocytes were counted under $\times 400$ magnification. Acute inflammatory responses were observed early after injection in all 4 areas, with no statistically significant differences among the groups. However, most inflammatory responses had subsided by week 8 after the injection.

\section{Filler absorption rate}

To measure the filler absorption rate, tissue slides were examined at high magnification at 4 and 8 weeks after filler injection. The normal saline was absorbed fastest at 4 weeks, and the fibrin glue was mostly absorbed at 8 weeks. The hyaluronic acid filler was slowly absorbed over time (Fig. 6). Compared to the hyaluronic acid filler, the PRFM filler using autologous plasma showed a marginal-
Table 1. Residual volume changes of the 4 types of filler

\begin{tabular}{lccc}
\hline \multirow{2}{*}{ Group } & \multicolumn{3}{c}{ Period } \\
\cline { 2 - 4 } & 2 weeks (mL) & 4 weeks $(\mathrm{mL})$ & 8 weeks $(\mathrm{mL})$ \\
\hline $\mathrm{A}^{\text {a) }}$ & & & \\
Vbowl & $0.1070 \pm 0.0002$ & $0.0210 \pm 0.0001$ & 0.0000 \\
VMRI & 0.1030 & 0.0190 & 0.0000 \\
Vavg & 0.1050 & 0.0200 & 0.0000 \\
B $^{\text {b) }}$ & & & \\
Vbowl & $0.2090 \pm 0.0001$ & $0.0940 \pm 0.0001$ & $0.0300 \pm 0.0004$ \\
VMRI & 0.2010 & 0.0860 & 0.0200 \\
Vavg & 0.2050 & 0.0900 & 0.0250 \\
$C^{c)}$ & & & \\
Vbowl & $0.8230 \pm 0.0001$ & $0.3540 \pm 0.0002$ & $0.1764 \pm 0.0001$ \\
VMRI & 0.8170 & 0.3460 & 0.1702 \\
Vavg & 0.8200 & 0.3500 & 0.1733 \\
$D^{d)}$ & & & 0.1402 \\
Vbowl & $0.7067 \pm 0.0001$ & $0.2700 \pm 0.0003$ & $0.1472 \pm 0.0001$ \\
VMRI & 0.6995 & 0.2650 & 0.1437 \\
Vavg & 0.7031 & 0.2675 & \\
\hline
\end{tabular}

$\mathrm{V}$, volume; MRI, magnetic resonance imaging; avg., average.

${ }^{a}$ Normal saline.

${ }^{b)}$ Fibrin glue.

${ }^{c l}$ Hyaluronic acid filler.

${ }^{d}$ Platelet-rich fibrin matrix filler.

ly higher but similar absorption rate. Based on the amount of the remaining filler at 8 weeks, the PRFM filler showed the same volume maintenance as hyaluronic acid (Fig. 7).

\section{DISCUSSION}

Various minimally invasive plastic surgery procedures are being performed using filler not only to remove aging-related wrinkles, but also for skin rejuvenation and in other procedures for aesthetic purposes [3]. Ongoing efforts have been made to determine the ideal filler for the correction of facial deformities, such as soft-tissue deficits, but there is still no substance that lasts permanently without adverse effects.

Recently, substances that are highly compatible with human tissues have been produced; one example is Restylane ${ }^{\circledR}$, which is a filler made up of hyaluronic acid. However, the greatest shortcoming of these synthetic fillers is that they are recognized as a foreign substance by the body, which can cause immune rejection or a granulomatous response. Several studies have reported that these fillers were biocompatible, but other studies have shown a risk of adverse reactions [3-6]. Moreover, when the area to be corrected is wider, a large amount of filler is required, and this presents limitations due to cost. To overcome the limitations of synthetic fillers, autologous tissue can be used to correct deficits, but graft surgery using autol- 


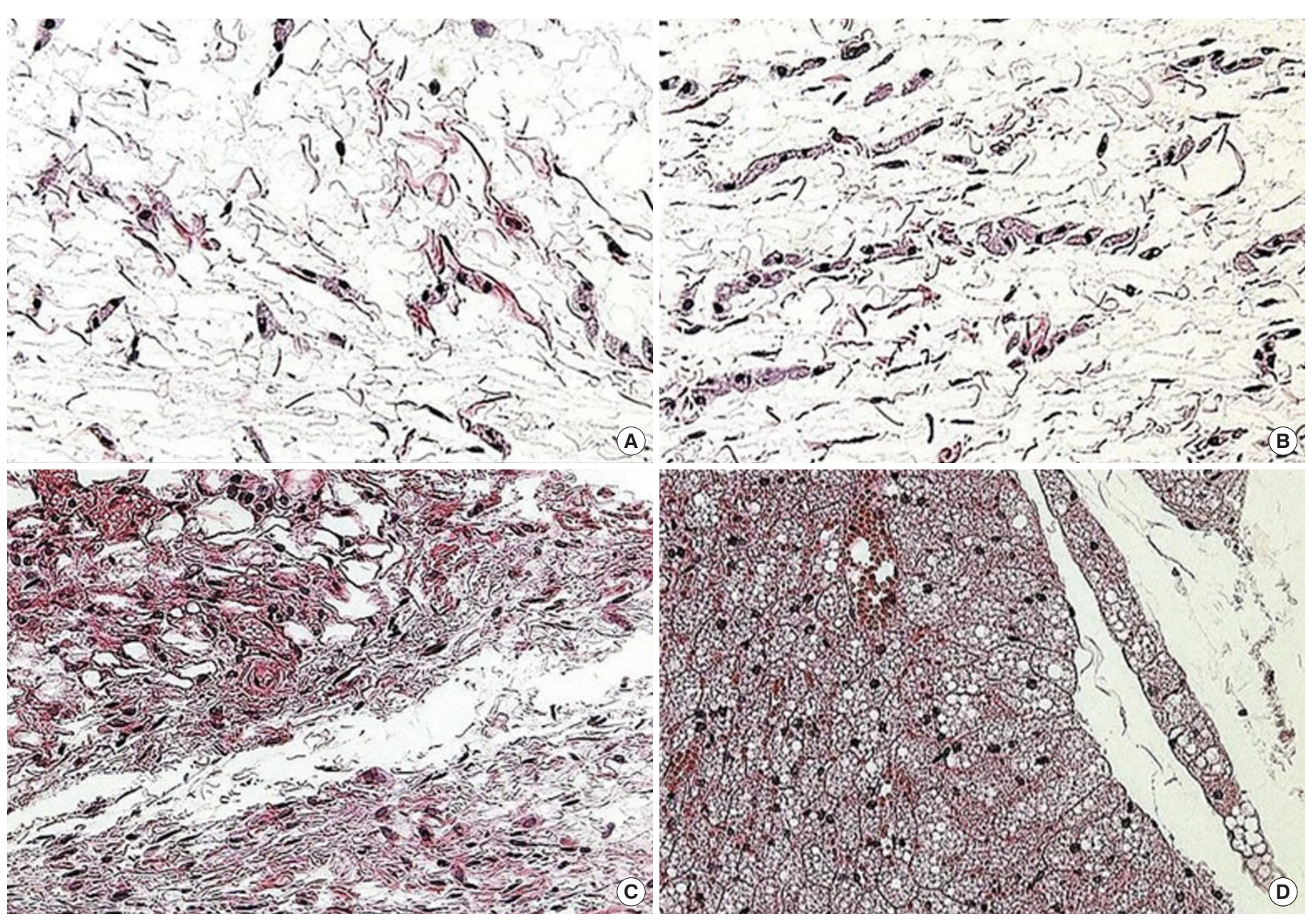

Fig. 6. Microphotographs at 4 weeks after the injection of 4 types of filler (hematoxylin and eosin stain [H\&E], $\times 200)$. (A) Normal saline, (B) fibrin glue, (C) hyaluronic acid filler, and (D) platelet-rich fibrin matrix (PRFM) filler. Normal saline and fibrin glue formed a loose, tidy filler material network between normal tissues. In contrast, the hyaluronic acid and PRFM fillers formed a denser, compact filler material network.

ogous tissue can cause complications, such as scarring, pain, and bleeding at the donor site. Therefore, PRFM filler from autologous plasma was used to achieve effective soft tissue augmentation without any hypersensitivity reaction while minimizing complications at the donor site.

Autologous platelets are an excellent source of growth factors due to their biological properties and endogenous origin, and they are the main source of growth factors that promote wound healing. Several studies have reported that PRP not only promoted early wound healing $[7,8]$, but also aided in the healing of diabetic ulcers [10].

In addition to the widespread use of PRP to promote wound healing, important clinical evidence can be observed in other areas of medicine. In addition to pathological issues, PRP is now also being successfully used for cosmetic purposes. The more substantial concentration of platelets compared with normal blood provides a unique source of growth factors. When PRP is injected into the target tissue in the dermis or subcutaneous layer, the platelets have an endogenous effect mediated by coagulation factors. Their activation causes significant degradation of the platelets, which in turn stimulates a chain of growth factors, such as platelet-derived growth factor, insulin-like growth factor, epidermal growth factor, and transforming growth factor beta. Activated platelets secrete numerous proteins, including adhesive glycoproteins such as fibrin, fibronectin, and vitronectin. Following subcutaneous injection, these proteins and growth factors interact with basal cells in the subcutaneous tissue, including fibroblasts, endothelial cells, and subcutaneous stem cells. After binding to specific cellular receptors, the glycoproteins and growth factors stimulate the production of extracellular matrix proteins, as well as the intracellular processes of cell proliferation, migration, and survival, and all these processes contribute to tissue rejuvenation [13].

We fabricated PRFM using Thrombo, Push-Man, and Air-Man kits. These kits are used for various orthopedic, vascular, and oral 


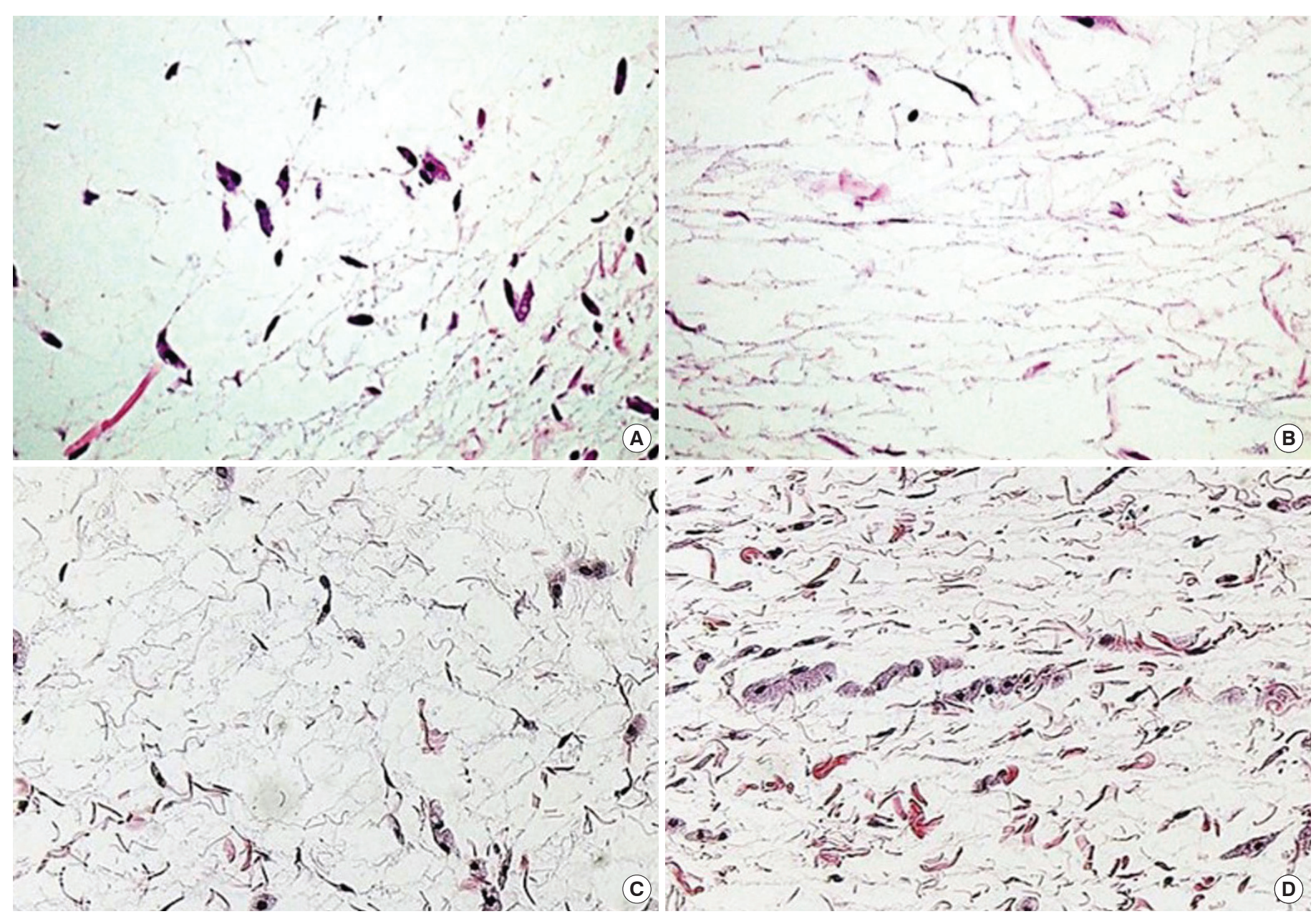

Fig. 7. Microphotographs at 8 weeks after injection of the 4 types of filler (hematoxylin and eosin stain [H\&E], $\times 200)$. (A) Normal saline, (B) fibrin glue, (C) hyaluronic acid filler, and (D) platelet-rich fibrin matrix (PRFM) filler. The normal saline had been absorbed completely, whereas a little fibrin glue remained. An abundant amount of the hyaluronic acid and PRFM fillers was observed.

and maxillofacial surgical procedures. In each field, PRFM promotes the healing process through angiogenesis in association with tissueappropriate cellular proliferation. The regeneration of both bone and soft tissue has been confirmed from in vitro and in vivo experiments. The topical application of PRFM is excellent for the treatment of refractory venous leg ulcers [14], and its clinical use in facial plastic surgery has shown outstanding outcomes in various studies $[15,16]$. PRFM not only contains various growth factors, but also produces no adverse effects, such as immune rejection. It does not have limitations in terms of economic accessibility, even for extensive corrections, and can be used effectively both for the soft tissue augmentation of facial deficits and for general filler applications, such as wrinkle correction $[17,18]$. Therefore, since PRFM is effective at restoring deficits, and the growth factors also stimulate regeneration by inducing proliferation of the surrounding skin, PRFM could enhance skin elasticity and correct shallow wrinkles. However, previous studies have reported conflicting opinions about the durability of PRFM filler compared with hyaluronic acid filler. Other studies were limited by only applying the treatment to patients in clinical settings. In the present study, we conducted an experiment involving nude mice to verify the efficacy of PRFM filler on soft tissue augmentation and volume maintenance.

During the 8-week study period, we observed pattern changes in nodules where 4 fillers had been injected. No signs of infection were noted, but histological observations showed an early inflammatory response for all 4 fillers; however, the acute inflammatory response was no longer present at 8 weeks. Compared with normal saline and fibrin glue, the hyaluronic acid and PRFM fillers showed better nodule formation and a more desirable change in shape for soft tissue augmentation. Volumetry was used to analyze the duration of the maintenance of the soft tissue augmentation effect. A superior augmentation effect was observed for the PRFM filler compared with fibrin glue and normal saline, and this effect was maintained significantly throughout the study period. Compared 
with the hyaluronic acid filler Restylane ${ }^{\circledast}$, which is currently used ubiquitously, PRFM filler showed a slightly higher absorption rate, but no difference was noted in the soft tissue augmentation effect or volume maintenance. Nevertheless, the 8-week duration is a limitation of this study, since fillers are used in contexts that require long-term durability. To overcome this limitation, a 6 to 12 -month longterm study and clinical trials are required. PRFM filler from autologous plasma is made up of the patient's own blood, which means that it produces no immune rejection. It has few economic limitations for extensive correction, contains various growth factors that enhance skin regeneration, and is effective at improving skin elasticity and correcting wrinkles [3,14-18].

In conclusion, the PRFM filler showed no statistically significant difference from the hyaluronic acid filler that is in current use in terms of durability during the 2-month study period, and its soft tissue augmentation effects were also the same. Therefore, PRFM filler using autologous plasma may be a good soft tissue filler for correcting facial deficits.

\section{PATIENT CONSENT}

Patients provided written consent for the use of their images.

\section{REFERENCES}

1. Roh NK, Kim MJ, Lee YW, et al. A split-face study of the effects of a stabilized hyaluronic acid-based gel of nonanimal origin for facial skin rejuvenation using a stamp-type multineedle injector: a randomized clinical trial. Plast Reconstr Surg 2016;137:809-16.

2. Dover JS, Rubin MG, Bhatia AC. Review of the efficacy, durability, and safety data of two nonanimal stabilized hyaluronic acid fillers from a prospective, randomized, comparative, multicenter study. Dermatol Surg 2009;35 Suppl 1:322-30; discussion 30-1.

3. Lowe NJ, Maxwell CA, Lowe P, et al. Hyaluronic acid skin fillers: adverse reactions and skin testing. J Am Acad Dermatol 2001;45:930-3.

4. Lupton JR, Alster TS. Cutaneous hypersensitivity reaction to injectable hyaluronic acid gel. Dermatol Surg 2000;26:135-7.

5. Patel VJ, Bruck MC, Katz BE. Hypersensitivity reaction to hyaluronic acid with negative skin testing. Plast Reconstr Surg 2006;117:92e-4e.

6. Glaich AS, Cohen JL, Goldberg LH. Injection necrosis of the glabella: protocol for prevention and treatment after use of dermal fillers. Dermatol Surg 2006;32:276-81.

7. Hom DB, Linzie BM, Huang TC. The healing effects of autologous platelet gel on acute human skin wounds. Arch Facial Plast Surg 2007; 9:174-83.

8. Kim JH, Park C, Park HM. Curative effect of autologous platelet-rich plasma on a large cutaneous lesion in a dog. Vet Dermatol 2009;20: 123-6.

9. Cho SW, Song KW, Rhie JW, et al. Engineered adipose tissue formation enhanced by basic fibroblast growth factor and a mechanically stable environment. Cell Transplant 2007;16:421-34.

10. Dougherty EJ. An evidence-based model comparing the cost-effectiveness of platelet-rich plasma gel to alternative therapies for patients with nonhealing diabetic foot ulcers. Adv Skin Wound Care 2008;21: 568-75.

11. Cho JM, Lee YH, Baek RM, et al. Effect of platelet-rich plasma on ultraviolet b-induced skin wrinkles in nude mice. J Plast Reconstr Aesthet Surg 2011;64:e31-9.

12. Yuksel EP, Sahin G, Aydin F, et al. Evaluation of effects of platelet-rich plasma on human facial skin. J Cosmet Laser Ther 2014;16:206-8.

13. Martínez-Zapata MJ, Martí-Carvajal A, Solà I, et al. Efficacy and safety of the use of autologous plasma rich in platelets for tissue regeneration: a systematic review. Transfusion (Paris) 2009;49:44-56.

14. O'Connell SM, Impeduglia T, Hessler K, et al. Autologous platelet-rich fibrin matrix as cell therapy in the healing of chronic lower-extremity ulcers. Wound Repair Regen 2008;16:749-56.

15. Sclafani AP, Saman M. Platelet-rich fibrin matrix for facial plastic surgery. Facial Plast Surg Clin North Am 2012;20:177-86, vi.

16. Sclafani AP. Safety, efficacy, and utility of platelet-rich fibrin matrix in facial plastic surgery. Arch Facial Plast Surg 2011;13:247-51.

17. Hui Q, Chang P, Guo B, et al. The clinical efficacy of autologous platelet-rich plasma combined with ultra-pulsed fractional CO2 laser therapy for facial rejuvenation. Rejuvenation Res 2017;20:25-31.

18. Ardakani MR, Moein HP, Beiraghdar M. Tangibility of platelet-rich fibrin matrix for nasolabial folds. Adv Biomed Res 2016;5:197. 